\title{
Frequência e perfil de resistência de Klebsiella spp. em um hospital universitário de Natal/RN durante 10 anos
}

\author{
Frequence and resistance profile of Klebsiella spp. isolates in a university hospital in Natal/RN during a ten-year period
}

Claudio Bruno Silva de Oliveira'; Valéria Cristina Ribeiro Dantas²; Renato Motta Neto ${ }^{3}$;

Paulo Roberto Medeiros de Azevedo ; Maria Celeste Nunes de Melo ${ }^{5}$

\section{unitermos \\ Resistência}

Hospital

Klebsiella spp.

ESBL

\section{resumo}

Introdução: As espécies de Klebsiella spp. podem causar vários tipos de infecções, principalmente hospitalares, e têm merecido destaque pelos seus variados e emergentes mecanismos de resistência. Objetivos: Determinar a frequência de isolamento e a caracterização do perfil de resistência de Klebsiella spp. em um hospital universitário durante um período de 10 anos e, ainda, avaliar a tendência para o crescimento dessa resistência. Material e método: Fez-se um estudo descritivo e retrospectivo a partir de dados coletados nos livros de registro do Laboratório de Microbiologia Clínica do hospital investigado, correspondentes ao período de janeiro de 1999 a dezembro de 2008. Resultado: A frequência de isolamento de Klebsiella spp. foi de 13,4\% com predominância em uroculturas $(56,4 \%)$. Houve aumento significativo na resistência para a maioria dos antimicrobianos testados ao longo do período analisado com tendência para o crescimento da mesma. Nesse período, isolou-se $23 \%$ de Klebsiella spp. com fenótipo produtor de betalactamases de amplo espectro (ESBL). Discussão: $\mathrm{O}$ isolamento de Klebsiella spp. resistente a antimicrobianos em amostras de origem clínica e a deteç̧ão da tendência do crescimento da resistência, inclusive às drogas de reserva terapêutica, são motivos de grande preocupação. Nesse hospital, a implantação de métodos de triagem e de confirmatórios para os mecanismos de resistência de Klebsiella spp. poderiam auxiliar no diagnóstico e no tratamento das infecções causadas por esse microrganismo. Conclusão: A tendência de crescimento na resistência aos antibióticos detectada neste estudo reforça a importância de monitoramentos contínuos. Estes elucidam características locais, orientando para melhores medidas de controle.

\section{abstract}

Introduction: Klebsiella spp. species can cause several infections, particularly nosocomial ones. Furthermore, its multiple emerging resistance mechanisms have been widely described. Objectives: To determine the isolation frequency and resistance profile of Klebsiella spp. at a university hospital during a ten-year period as well as to assess the increase in its resistance. Material and method: A retrospective and descriptive study was carried out based on data collected from the record books of the Laboratory of Clinical Microbiology of the investigated Hospital from January 1999 to December 2008. Results: The isolation frequency of Klebsiella spp. was $13.4 \%$, predominantly in urine cultures (56.4\%). There was a significant increase in resistance to most antimicrobials tested over the analyzed period; $23 \%$ of Klebsiella spp. with ESBL phenotype was isolated over this period. Discussion: Multi-resistant Klebsiella spp. isolates from clinical samples as well as its growing trend in resistance mechanisms, including to reserve drugs, are cause for great concern. The implementation of screening and confirmatory methods of bacterial resistance could aid in the diagnosis and treatment of infections caused by this microorganism. Conclusion: The increase in resistance to antibiotics reinforces the importance of continuous monitoring, which elucidates local characteristics and allows more suitable control measures. key words

Resistance

Hospital

Klebsiella spp.

ESBL

\footnotetext{
1. Biomédico.

2. Mestre em Ciências Farmacêuticas; professora da Universidade Potiguar.

3. Doutor em Cirurgia pela Universidade Federal do Ceará (UFC); professor adjunto da Universidade Federal do Rio Grande do Norte (UFRN).

4. Doutor em Ciências Sociais pela UFRN; professor adjunto da UFRN.

5. Doutor em Ciências (Microbiologia) pela Universidade Federal do Rio de Janeiro (UFR)); professor adjunto da UFRN.
} 


\section{Introdução}

Atualmente, a resistência bacteriana se configura em um grande problema de saúde pública e é responsável pelo aumento significativo na morbidade e na mortalidade dos pacientes ${ }^{(1)}$. Essa resistência é comumente diagnosticada nos laboratórios de microbiologia clínica pelo método de disco-difusão, seguindo critérios estabelecidos pelo Clinical and Laboratory Standards Institute (CLSI), o qual revê e, eventualmente, atualiza anualmente esses critérios. Um importante grupo de microrganismos, reconhecido pelos seus diversos mecanismos de resistência, é a família Enterobacteriaceae. Esse grupo também tem merecido atenção especial nos últimos anos por estar entre os microrganismos mais isolados na rotina dos laboratórios de microbiologia clínica ${ }^{(22)}$. Nessa família a produção de betalactamases é um dos principais mecanismos de resistência, agindo contra os fármacos betalactâmicos, amplamente utilizados no combate a esse grupo de microrganismos ${ }^{(15)}$. $\mathrm{Na}$ família Enterobacteriaceae, um dos fundamentais patógenos para humanos, com conhecidos e potentes mecanismos de resistência, é o gênero Klebsiella spp. (7, 22). As espécies de Klebsiella spp. são causadoras de infecções em unidades de terapia intensiva (UTIs), onde afetam pacientes imunocomprometidos, que estão sujeitos a inúmeros fatores de risco, como administração de grande quantidade de antibióticos, doenças crônicas, procedimentos invasivos e tratamentos imunossupressores $^{(1,7)}$. No ambiente hospitalar, um dos mecanismos de resistência mais prevalente é a produção de betalactamases. Estas degradam os antimicrobianos belalactâmicos, que correspondem a uma classe amplamente empregada no tratamento de infecções graves. São exemplos desses fármacos as cefalosporinas de amplo espectro (ceftazidima, cefotaxima, cefepima) e os carbapenêmicos (imipenem, meropenem e ertapenem) ${ }^{(15)}$. No gênero Klebsiella spp., um importante mecanismo de resistência é proporcionado pelas betalactamases de amplo espectro (ESBL) ${ }^{(13)}$. Essas enzimas, codificadas por plasmídeos e amplamente distribuídas entre as enterobactérias (podendo vir acompanhadas de genes de resistência a aminoglicosídeos e cloranfenicol), são resultantes da mutação do gene da betalactamase comum, como TEM-1, TEM-2 e SHV-1 ${ }^{(9,11)}$, e têm capacidade de hidrolisar uma ampla variedade de penicilinas e cefalosporinas de terceira geração ${ }^{(7,23)}$, as quais foram originalmente desenvolvidas como drogas capazes de vencer a resistência bacteriana conferida pelas betalactamases comuns.
No momento, são quatro as principais betalactamases de interesse clínico e que apresentam espectro de ação distinto sobre os betalactâmicos ${ }^{(15)}$ :

- ESBL;

- Klebsiella pneumoniae carbapenemase (KPC);

- metalobetalactamase (MBL);

- betalactamase classe C (AmpC).

Desde a descoberta dos primeiros microrganismos produtores de ESBL, no início da década de 1980, essa bactéria adquiriu resistência a muitas outras classes de fármacos, como os aminoglicosídeos, e até mesmo aos carbapenêmicos, tidos como principais drogas no combate ao microrganismo, devido a sua estabilidade diante da atividade hidrolítica da ESBL e também pela capacidade de penetrar na bactéria ${ }^{(23)}$. A resistência aos carbapenêmicos é conferida por carbapenemases, como KPC e metalobetalactamases, pela perda de proteínas da membrana externa e por outros mecanismos ${ }^{(20)}$. Diante das limitações terapêuticas provocadas pelos mecanismos de resistência, diversos grupos de pesquisa vêm acompanhando a distribuição e o perfil de suscetibilidade do gênero Klebsiella spp. ao redor do mundo, com o intuito de acompanhar possíveis surtos e montar o perfil de sensibilidade em diferentes regiões ${ }^{(7,22,26)}$. Um estudo de prevalência realizado na Espanha e no Reino Unido mostrou que a espécie Klebsiella pneumoniae, produzindo ESBL, é responsável por cerca de $26,8 \%$ das bacteremias comunitárias ${ }^{(4)}$. Na Turquia, uma pesquisa em 56 UTIs demonstrou prevalência de 16,1\% para o gênero Klebsiella spp. ${ }^{(8)}$. Em um hospital universitário de Taiwan, verificou-se aumento de $10 \%$ para $55 \%$ de $K$. pneumoniae produtora de $\mathrm{ESBL}^{(28)}$. Na cidade de Nova York, detectou-se prevalência de $17 \%$ de $K$. pneumoniae entre os microrganismos Gram-negativos isolados em 15 hospitais ${ }^{(21)}$. Em estudos brasileiros, a prevalência de Klebsiella spp. varia de $13,4 \%$ a $15 \%^{(12,14)}$. Nessas pesquisas, esse gênero aparece como um dos principais envolvidos em infecções do trato urinário ${ }^{(12,19)}$ com importante destaque nas infecções em UTIs ${ }^{(16)}$ e nos isolados de ponta de cateter e aspirados traqueais ${ }^{(14)}$. A mudança no perfil de sensibilidade aos antimicrobianos de cepas de Klebsiella spp., bem como a existência de relatos crescentes de surtos hospitalares causados por esses agentes, justifica o monitoramento desse patógeno no ambiente nosocomial. Porém, a variação no espectro de ação das enzimas faz com que a padronização de um teste, viável, de sensibilidade in vitro para detecção fenotípica de cepas produtoras de betalactamases seja dificultada ${ }^{(29)}$. Contudo, o CLSI sugere alguns métodos fenotípicos para screening 
(avaliação da sensibilidade a aztreonam, ceftazidima, ceftriaxona e cefotaxima) e para confirmação da produção de ESBL (comparação dos halos de inibição para ceftazidima e cefotaxima, com e sem associação ao ácido clavulânico) que podem ser incorporados na rotina laboratorial e, dessa forma, contribuir para a elaboração de um diagnóstico correto ${ }^{(3,19)}$. O reconhecimento do gênero Klebsiella spp. como importante patógeno envolvido em infecções, principalmente hospitalares, e as variações regionais no perfil de resistência aos antibióticos, além do conhecimento de seus emergentes mecanismos de resistência, são fatores que reforçam a necessidade de se conhecer as características locais desse gênero, assim como o processo de evolução de sua resistência. Esse conhecimento poderá contribuir para o melhor direcionamento e uso de antimicrobianos.

\section{Objetivos}

O presente estudo teve como objetivos determinar a frequência de isolamento, a caracterização do perfil de resistência e, ainda, avaliar a tendência da resistência para o crescimento em Klebsiella spp. provenientes de um hospital universitário localizado na cidade de Natal/ $\mathrm{RN}$, correspondentes ao período de janeiro de 1999 a dezembro de 2008.

\section{Material e método}

Foi feito um estudo descritivo e retrospectivo a partir de dados secundários coletados nos livros de registro do Laboratório de Microbiologia Clínica de um hospital universitário localizado na cidade de Natal/RN, correspondentes ao período de janeiro de 1999 a dezembro de 2008. Os dados analisados a partir do livro de registro foram o sítio de origem das amostras, a resistência aos antimicrobianos, assim como o possível fenótipo produtor de ESBL. Incluiu-se na pesquisa apenas as amostras não duplicadas. O critério utilizado pelo laboratório para determinação da resistência aos antimicrobianos foi o estabelecido pelo National Committee for Clinical Laboratory Standards (NCCLS), o qual, a partir de 2005, passou a denominar-se CLSI. Amicacina, ciprofloxacino, cefotaxima, ceftriaxona, ceftazidima, cefoxitina, aztreonam, imipenem, ertapenem, piperacilina/tazobactam foram os antimicrobianos avaliados neste estudo. Para a classificação de Klebsiella spp. com fenótipo produtor de ESBL utilizamos como critério o estabelecido pelo CLSI 2009(3), em que classifica como produtoras de ESBL as cepas que apresentam resistência simultaneamente ao monobactâmico aztreonam, assim como às cefalosporinas de terceira geração (ceftazidima, ceftriaxona e cefotaxima), pelo método de disco-difusão. A análise relativa de determinação da tendência para o crescimento da resistência foi realizada por meio do método de regressão linear simples, sendo a variável dependente a resistência e a independente, o tempo. Mediante esse modelo, testou-se a hipótese de que o coeficiente da variável independente é zero ou não. Rejeitando-se a hipótese nula, conclui-se pela existência de tendência nas séries dos dados. O processamento dos dados foi realizado com auxílio do software estatístico R, de domínio público, na versão 2.8.1. O valor de $p<0,05$ foi considerado como estatisticamente significativo.

\section{Resultado}

Durante o período analisado, o Laboratório de Microbiologia Clínica registrou 6.401 exames positivos para bactérias, dos quais o gênero Klebsiella spp. apresentou uma frequência de isolamento de $13,4 \%$. Com relação ao sítio de isolamento, o maior número de isolados positivos para esse microrganismo foi encontrado em uroculturas, seguido de secreções, hemoculturas e pontas de cateter (Tabela 1).

Valores absolutos e percentuais de Klebsiella spp. distribuídos por sítios de isolamento em um hospital universitário na cidade de Natal/RN,

Tabela 1 no período de 1999 a 2008

\begin{tabular}{lcc}
\hline Amostra biológica & N & $\%$ \\
Urina & 484 & 56,4 \\
Secreções & 237 & 27,6 \\
Sangue & 112 & 13 \\
Ponta de cateter & 25 & 2,9 \\
Total & 858 & 100 \\
\hline
\end{tabular}

A Tabela 2 mostra a variação anual e a tendência para o crescimento da resistência aos antimicrobianos de Klebsiella spp. durante o período analisado. Pode-se constatar que praticamente todos os antimicrobianos testados apresentaram aumento da resistência aos agentes antimicrobianos com o passar dos anos. No caso da cefoxitina e do imipenem não ocorreram alterações significativas. As 
maiores taxas na evolução da resistência foram observadas com piperacilina/tazobactam, ciprofloxacino, cefalosporinas de terceira geração e aztreonam.

\section{Variação anual e tendência para \\ o crescimento da resistência \\ aos antimicrobianos de \\ Klebsiella spp. em um hospital \\ universitário na cidade de Natal/RN,}

Tabela 2 no período de 1999 a 2008

\begin{tabular}{|c|c|c|}
\hline Antibiótico & $\begin{array}{c}\text { Mudança em \%l } \\
\text { ano (* } p \text { - valor) }\end{array}$ & Tendência \\
\hline Amicacina & $3,36(0,0081)$ & Crescente \\
\hline Ciprofloxacino & $6,31(0)$ & Crescente \\
\hline Cefotaxima & 7,77 (0) & Crescente \\
\hline Ceftriaxona & $3,92(0,0105)$ & Crescente \\
\hline Ceftazidima & $4,06(0,0044)$ & Crescente \\
\hline Cefoxitina & $-1,09(0,2810)$ & Estável \\
\hline Aztreonam & $4,39(0,0104)$ & Crescente \\
\hline Imipenem & $0,12(0,7830)$ & Estável \\
\hline Ertapenem & $3,63(0,0104)$ & Crescente \\
\hline $\begin{array}{l}\text { Piperacilina/ } \\
\text { tazobactam }\end{array}$ & $8,84(0,0023)$ & Crescente \\
\hline
\end{tabular}

* 0 valor de $p<0,05$ foi considerado estatisticamente significativo.

$\mathrm{Na}$ Tabela 3 pode-se observar o número de amostras submetidas ao teste de triagem para ESBL, bem como o número de amostras de Klebsiella spp. com o perfil sugestivo do fenótipo produtor de ESBL, relacionado com os três intervalos de tempo e o período de 10 anos da pesquisa.

Número de amostras submetidas ao teste de triagem para ESBL; valores percentuais de cepas

de Klebsiella spp. com fenótipo produtor de ESBL, relacionado com os três intervalos de tempo e com 0

Tabela 3 período total da pesquisa

\begin{tabular}{lcc}
\hline Período & $\begin{array}{c}\text { Amostras testadas para } \\
\text { a produção de ESBL }\end{array}$ & $\begin{array}{c}\text { Fenótipo } \\
\text { ESBL }\end{array}$ \\
\hline $1999-2002$ & 72 & $16,7 \%$ \\
$2003-2005$ & 122 & $28,57 \%$ \\
$2006-2008$ & 4 & $75 \%$ \\
Total & 198 & $23,07 \%$ \\
\hline
\end{tabular}

ESBL: betalactamases de amplo espectro.

\section{Discussão}

A presença do gênero Klebsiella spp. em amostras de origem clínica implica em grande preocupação, uma vez que se sabe que esse microrganismo apresenta diversos mecanismos de resistência e pode estar envolvido em inúmeras patologias, de brandas a graves, tendo como consequência o óbito ${ }^{(10,15)}$. A frequência de isolamento encontrada neste estudo é similar à de outros estudos no Brasil ${ }^{(12,14)}$. Dados similares também foram observados em pesquisas internacionais $(6,25,26)$. Os dados relacionados com o sítio de isolamento também corroboram os de outros estudos, que mostram o gênero Klebsiella spp. como importante patógeno envolvido em infecções do trato urinário( 7,12$)$. A análise de tendência da evolução da resistência permitiu verificar que a maioria dos antibióticos testados, com exceção da cefoxitina e do imipenem, apresentou um perfil de resistência com tendência estatisticamente significativa de crescimento com o passar dos anos, desde que mantidas as atuais práticas de administração de antimicrobianos. A estabilidade na resistência da cefoxitina costuma ser creditada ao fato desta não ser rotineiramente utilizada na prática clínica para o tratamento de infecções por Klebsiella spp. ${ }^{(19)}$. Com relação ao imipenem, a tendência para a estabilidade pode ser resultado do uso mais amplo de outra droga da mesma classe, como o ertapenem, o que provavelmente contribuiu para uma taxa mais alta na resistência a esse antimicrobiano, bem como para a tendência de crescimento na resistência do mesmo. A resistência à família dos carbapenêmicos tem sido detectada ${ }^{(5,29)}$ e recebido grande atenção nos últimos anos pelo fato desses fármacos serem a classe de escoIha no tratamento de Klebsiella spp. produtora de ESBL, e também pelos seus emergentes mecanismos de resistên$\mathrm{cia}^{(2,10,20)}$. Observou-se que, dependendo da metodologia empregada no teste de suscetibilidade, pode haver diferentes perfis de resistência dentro da mesma classe de antibióticos(2), o que significa que a bactéria pode utilizar mecanismos de resistência diferentes para os carbapenêmicos. Normalmente, o gênero Klebsiella spp. recorre à alteração de porinas como mecanismo de resistência ao imipenem, que devido ao seu maior tamanho tem mais dificuldade em penetrar no microrganismo (esse mecanismo também pode conferir baixos níveis de resistência ao meropenem), e também pode se valer de outros métodos de resistência, como as bombas de 
efluxo ${ }^{(18)}$. Já para o ertapenem, os principais mecanismos de resistência são baseados na produção de carbapenemases e na perda de proteínas da membrana externa ${ }^{(24,27)}$. Nesse período, alguns dos fármacos como a piperacilina/ tazobactam e o ciprofloxacino apresentaram elevadas variações anuais na porcentagem de resistência, o que pode ser reflexo do amplo uso dos mesmos. Esse é um dado preocupante, uma vez que os antibióticos analisados nesta pesquisa estão entre as principais classes (aminoglicosídeos, quinolonas, cefalosporinas de terceira geração, carbapenêmicos e penicilina + inibidor de betalactamase) utilizadas no tratamento da bactéria em questão ${ }^{(10,20)}$. A ampla resistência apresentada comprova a evolução e a aquisição de diferentes mecanismos de resistência pelo microrganismo, tornando, desse modo, cada vez mais limitados os esquemas terapêuticos. As altas taxas de resistência às cefalosporinas de terceira geração e ao aztreonam, observadas neste estudo, apresentam perfil sugestivo de Klebsiella spp. produtora de $E S B L$, porém o pequeno número de isolados submetidos aos testes de triagem nos impede de obter dados mais fidedignos da real produção dessa enzima. Contudo, não podemos deixar de destacar um aumento importante na frequência de isolamento de Klebsiella spp. com fenótipo ESBL durante o tempo da pesquisa. Esse aumento pode ser reflexo do grande uso de cefalosporinas de terceira geração. Entre os fatores limitantes na análise dos dados, destacam-se a mudança dos antibióticos testados ao longo dos anos (alguns foram incorporados na rotina do laboratório, enquanto outros deixaram de ser utilizados) e o fato de ser uma análise retrospectiva baseada apenas nos livros de registros, de modo que alguns dados relativos à coleta e ao processamento das amostras não puderam ser acompanhados. No entanto, apesar dos aspectos limitantes envolvidos, estudos desse tipo são importantes por elucidarem características acerca da resistência no passado, permitindo analisar variações e tendências do processo de resistência ao longo dos anos. O conhecimento da resistência bacteriana, bem como seus mecanismos de ocorrência e estratégias de prevenção e controle, constituem fortes fundamentos para reflexões e revisões de conduta e protocolo. Acredita-se que somente a partir de tais atitudes seja possível investir no processo de controle da disseminação dos microrganismos multirresistentes, principalmente quando se observam registros da progressiva evolução da resistência bacteriana, presente não só no contexto hospitalar, mas também no comunitário(17). Entre essas medidas, podem-se destacar as pesquisas por novas técnicas de diagnóstico, além da utilização das atuais técnicas laboratoriais padronizadas, eficazes e recomendadas pelo CLSI para a detecção de amostras de $K$. pneumoniae produtoras de $\mathrm{ESBL}^{(23)}$, assim como outras metodologias que visam o monitoramento constante da evolução de outros mecanismos de resistência. Tais medidas, somadas ao controle rigoroso na utilização de antimicrobianos, poderão inibir ou limitar o aparecimento das cepas e evitar a perda gradativa de antibióticos potentes do arsenal terapêutico. É nesse sentido que o presente estudo veio suprir a necessidade de um conhecimento mais aprofundado da frequência e do perfil de suscetibilidade aos antimicrobianos do gênero Klebsiella spp. no hospital pesquisado na cidade de Natal/RN, apresentando a evolução da resistência dessa bactéria com o passar dos anos. Contudo, a análise dos resultados obtidos neste estudo não permite estender as conclusões aos demais hospitais da referida cidade, visto que as frequências de isolamento podem apresentar variações entre os diversos hospitais, uma vez que elas ocorrem pelas diferentes políticas de uso de antimicrobianos e pelo perfil de atendimento do hospital. Esta pesquisa (abrangendo um longo intervalo de tempo) é uma contribuição inédita e significativa para o esclarecimento de características locais desse gênero e serve como passo inicial para que mais estudos e pesquisas relacionadas sejam desenvolvidos.

\section{Conclusão}

Nesse estudo foi encontrada frequência de isolamento de Klebsiella spp. concordante com a literatura. Entre esses isolados, observou-se tendência de crescimento na resistência na maioria dos antibióticos testados, o que evidencia a necessidade de novos estudos para monitorar tanto a evolução da resistência quanto o surgimento de novos mecanismos de resistência. Os dados observados nesta pesquisa apresentam perfil sugestivo de Klebsiella spp. produtora de ESBL, entretanto, o pequeno número de isolados submetidos aos testes de triagem nos impede de obter dados mais fidedignos da real produção dessa enzima. Sugere-se a implantação desses testes para que se possa determinar a dimensão dessa problemática e, assim, poder traçar as medidas necessárias para o controle desse microrganismo. 


\section{Referências}

1. ALBRICH, W. C. et al. Drug resistance in intensive care units. Infection, v. 27, p. S19-23, 1999.

2. BRATU, S. et al. Emergence of KPC-possessing Klebsiella pneumoniae in Brooklyn, New York: epidemiology and decommendations for detection. Antimicrob Agents Chemother, v. 49, n. 7, p. 3018-20, 2005.

3. CLINICAL AND LABORATORY STANDARDS INSTITUTE. Performance standards for antimicrobial susceptibility testing: nineteenth informational supplement. M100-S19. Clinical and Laboratory Standards Institute, Wayne, PA, 2009.

4. COQUE, T. M.; BAQUERO, F.; CANTON, R. Increasing prevalence of ESBL-producing Enterobacteriaceae in Europe. Eurosurveillance, v. 13, n. 47, 2008.

5. DIENSTMANN, R. et al. Avaliação fenotípica da enzima Klebsiella pneumoniae carbapenemase (KPC) em Enterobacteriaceae de ambiente hospitalar. J Bras Patol Med Lab, v. 46, n. 1, p. 23-7, 2010.

6. DIMITROV, T. S. et al. Etiology and antibiotic susceptibility patterns of community-acquired urinary tract infections in a Kuwait hospital. Med Princ Pract, v. 13, p. 334-9, 2004.

7. EARSS - European Antimicrobial Resistance Surveillance System. EARSS annual report 2007. 2008.

8. ESEN, S.; LEBLEBICIOGLU, H. Prevalence of nosocomial infections at intensive care units in Turkey: a multicentre 1-day point prevalence study. Scand J Infect Dis, v. 36, n. 2, p.144-8, 2004.

9. GNIADKOWSKI, M. Evolution and epidemiology of extended spectrum beta-lactamases (ESBLs) and ESBLproducing microorganisms. Clin Microbiol Infect, v. 7, p. 597-608, 2001.

10. GUPTA, A. et al. Extended Spectrum $\beta$ Lactamaseproducing Klebsiella pneumoniae infections: a review of the literature. J Perinatology, v. 23, p. 439-43, 2003.

11. JAIN, A.; MONDAL, R. TEM \& SHV genes in extended spectrum $\beta$-lactamase producing Klebsiella species $\&$ their antimicrobial resistance pattern. Indian J Med Res, v. 128, p 759-64, 2008.

12. KAZMIRCZAK, A.; GIOVELLI, F. H.; GOULART, L. S. Caracterização das Infecções do trato urinário diagnosticadas no município de Guarani das MissõesRS. Rev Bras Anal Clin, v. 37, n. 4, p. 205-7, 2005.

13. KNOTHE, $\mathrm{H}$. et al. Transferable resistance to cefotaxime, cefoxitin, cefamandole and cefuroxime in clinical isolates of Klebsiella pneumoniae and Serratia Marcescens. Infection, v. 11, n. 6, 1983.

14. MENEZES, E. A. et al. Freqüência e percentual de suscetibilidade de bactérias isoladas em pacientes atendidos na unidade de terapia intensiva do Hospital Geral de Fortaleza. J Bras Patol Med Lab, v. 43, n. 3, p. 149-55, 2007.

15. MEYER, G.; PICOLI, S. U. Fenótipos de betalactamases em Klebsiella pneumoniae de hospital de emergência de Porto Alegre. J Bras Patol Med Lab, v. 47, n. 1, p. 25-31, 2011.

16. MOURA, M. E. B. et al. Infecção hospitalar: estudo de prevalência em um hospital público de ensino.
Rev Bras Enferm, Brasília, v. 60, n. 4, p. 416-21, 2007.

17. OLIVEIRA, A. C.; SILVA, R. S. Desafios do cuidar em saúde frente à resistência bacteriana: uma revisão. Rev Eletr Enf, v. 10, n. 1, p. 189-97, 2008.

18. PAl, $\mathrm{H}$. et al. Carbapenem resistance mechanisms in Pseudomonas aeruginosa clinical isolates. Antimicrob Agents Chemother, p. 480-4, 2001.

19. PASTA, A. A. C. et al. Prevalência e perfil de susceptibilidade antimicrobiana em cepas de Klebsiella pneumoniae produtoras de $\beta$-lactamases de espectro estendido (ESBL), isoladas de pacientes do Hospital Universitário/ UEL. Rev Bras Anal Clin, v. 40, n. 2, p. 137-41, 2008.

20. PATERSON, D. L. Resistance in Gram-negative bacteria: Enterobacteriaceae. Americ J Med, v. 119, n. 6A, p. 20-8, 2006.

21. SAURINA, G. et al. Antimicrobial resistance in Enterobacteriaceae in Brooklyn, NY: epidemiology and relation to antibiotic usage patterns. J Antimicrob Chemother, v. 45, p. 895-8, 2000.

22. SOULI, M.; GALANI, I.; GIAMARELLOU, H. Emergence of extensively drug-resistant and pandrug-resistant Gram-negative bacilli in Europe. Eurosurveillance, v. 13, n. 47, 2008.

23. STURENBURG, E.; MACK, D. Extended-spectrum betalactamases: implications for the clinical microbiology laboratory, therapy, and infection control. J Infect, v. 47, n. 4, p. 273-95, 2003.

24. SZABÓ, D. et al. Outer membrane protein changes and efflux pump expression together may confer resistance to ertapenem in Enterobacter cloacae. Antimicrob Agents Chemother, p. 2833-5, 2006.

25. TONKIC, M.; BARISIC, I. G.; PUNDA-POLIC, V. Prevalence and antimicrobial resistance of extended-spectrum $\beta$-lactamases-producing Escherichia coliand Klebsiella pneumoniae strains isolated in a university hospital in Split, Croatia. Int Microbiol, v. 8, p.119-24, 2005.

26. TRUCCO, O. et al. Rede de vigilância de resistência antimicrobiana PRONARES. Informe primeiro semestre 2001. Rev Chil Infect, v. 19, Supl. 2, p. 140-8, 2002.

27. WOODFORD, N. et al. Ertapenem resistance among Klebsiella and Enterobacter submitted in the UK to a reference laboratory. Internat J Antimicrob Agents, v. 29, p. 456-9, 2007.

28. WU, J. J. et al. Prevalence and characteristics of ertapenem-resistant Klebsiella pneumoniae isolates in a Taiwanese university hospital. Microb Drug Resist, v. 17, n. 2, p. 259-66, 2011.

29. ZANOL, F. M.; PICOLI, S. U.; MORSCH, F. Detecção fenotípica de metaloßlactamase em isolados clínicos de Pseudomonas aeruginosa de hospitais de Caxias do Sul. J Bras Patol Med Lab, v. 46, n. 4, p. 309-14, 2010.

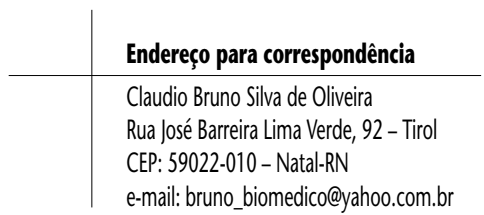

